\title{
Post-Traumatic Seizures: A Deep-Dive Into Pathogenesis
}

Fatima Anwer ${ }^{1}$, Federico Oliveri ${ }^{2}$, Fotios Kakargias ${ }^{1}$, Priyanka Panday ${ }^{1}$, Ana P. Arcia Franchini ${ }^{1}$, Beshoy Iskander $^{3}$, Pousette Hamid ${ }^{4}$

1. Research, California Institute of Behavioral Neurosciences \& Psychology, Fairfield, USA 2. Cardiology, California Institute of Behavioral Neurosciences \& Psychology, Fairfield, USA 3. Internal Medicine, California Institute of Behavioral Neurosciences \& Psychology, Fairfield, USA 4. Neurology, California Institute of Behavioral Neurosciences \& Psychology, Fairfield, USA

Corresponding author: Fatima Anwer, fatimaanwar47@gmail.com

\section{Abstract}

Post-traumatic seizures (PTS) have become an emerging challenge for neurologists worldwide with the rise of brain injuries. Trauma can lead to various outcomes, ranging from naive spasms to debilitating posttraumatic epilepsy (PTE). In this article, we will explore the pathogenesis of convulsions following a concussion. We will look at multiple studies to explain the various structural, metabolic, and inflammatory changes leading to seizures. Additionally, we will explore the association between severity and location of injury and PTE. PTE's pathophysiology is not entirely implicit, and we are still in the dark as to which antiepileptic drugs will be useful in circumventing these attacks. The purpose of this narrative review is to explain the post-traumatic brain changes in detail so that such attacks can be either thwarted or treated more resourcefully in the future.

Received 02/27/2021

Review began 03/31/2021 Review ended 04/09/2021 Published 04/10/2021

(c) Copyright 2021

Anwer et al. This is an open access article distributed under the terms of the Creative Commons Attribution License CC-BY 4.0., which permits unrestricted use, distribution, and reproduction in any medium, provided the original author and source are credited.
Categories: Internal Medicine, Neurology, Pathology

Keywords: trauma and epilepsy, brain injury and seizure, head injury and epilepsy, post-traumatic epilepsy, motor vehicle accident and epilepsy, post-traumatic seizure

\section{Introduction And Background}

Traumatic brain injury (TBI) has been emerging as a significant concern recently due to the rising number of cases leading to long-term disability, impacting the healthcare system [1]. TBI can lead to numerous adverse effects ranging from simple seizures to debilitating chronic epilepsy [2].

The risk of developing post-traumatic epilepsy (PTE) varies depending on the site of impact and severity of the injury. It is $4.4 \%$ mild TBI, $7.6 \%$ for moderate, and $13.6 \%$ after severe brain injury [3]. The risk is more in patients with a severe injury, older age, and those suffering from early seizures. Additionally, the risk is also higher among individuals who do not receive anti-epileptic medications [2].

\section{Classification of post-traumatic seizures}

Post-traumatic seizures (PTS) have been classified based on the time of onset after injury (Figure 1). The ones occurring within 24 hours are called "immediate." "Early seizures" appear between 24 hours and one week. Those after one week are "late seizures" [2]. In some cases, repeated unprovoked seizures one week after trauma traditionally referred to as "PTE" might occur. These are different from acute episodes $(<1$ week), which are mostly triggered. The PTE can lead to a detrimental effect on the quality of life [4]. 


\section{Classification of Post-Traumatic Seizures}

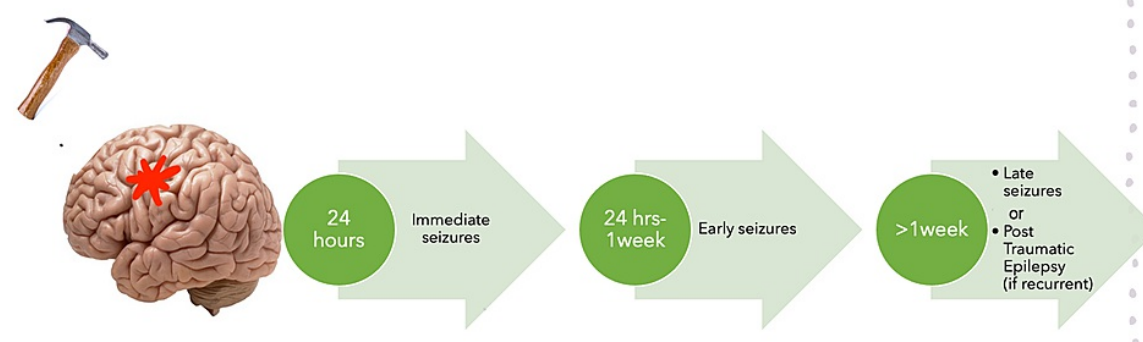

FIGURE 1: Classification of post-traumatic seizures.

\section{Pathogenesis of post-traumatic seizures}

Multiple mechanisms have been explained in the past that led to altered brain activity provoking seizures, including increased inflammatory markers, altered blood-brain barrier, changes in astrocytes, and glucose metabolism dysregulation [4-7].

\section{Role of Proteins}

Purines play a vital role in the pathogenesis of PTE. The upregulation of urate and decline in adenosine is also a culprit. Additionally, changes in the 2',3' Cyclic adenosine monophosphate pathway lowers the PTE's threshold [8]. Repeated brain injuries, despite the severity, can disturb the normal astrocyte response and cause scar formation. These changes, in turn, decrease Glial fibrillary acid protein expression and downregulate homeostatic proteins [9].

\section{The Astrocytes Controversy}

The role of astrocytes is still controversial. Some studies show that they protect the healthy brain by forming scar and separating the damaged area. On the other hand, some depict the same fibrosis spot as the focus for the seizure's origin $[5,9]$.

\section{Blood-Brain Barrier}

The blood-brain barrier (BBB) guards the neurons against the toxins in the blood and allows very selective molecules to pass through while preventing others. The main component of BBB are endothelial cells with the tight junction and astrocytes end feet and pericytes. Tomkins et al. studied the role of BBB leakiness in PTE. They followed 38 patients for two years after traumatic brain injury and observed the changes in BBB. Those who developed PTE had a greater BBB disruption area than those who recovered without any seizure attacks [10].

\section{Role of Cytokines}

Cytokines play a crucial role in the pathogenesis of PTE. Tumour necrosis factor-alpha (TNF- $\alpha$ ) disrupts BBB leading to the entry of leukocytes and causes neuronal degeneration. IL-6 plays a similar part. High mobility group box protein 1 (HMGB1) released by dying cells is also ictogenic. The macrophages, entering the brain after injury, emit chemokine ligand 2 that is ictogenic and causes further damage by recruiting more macrophages [4].

Genetics and Neuroplasticity

Neuroplasticity, the changes in neurons in response to trauma, play an essential role in PTE's pathogenesis, especially temporal lobe epilepsy [11]. Patients who experience epilepsy after injury appear to have poor functionality [12]. Certain genetic factors increase the probability of PTE, like the glutamate transporter gene mutations [13]. In around one-third of the scenarios, PTE is resistant to medication, so a vast understanding of the modifications is vital to reduce PTE occurrence [4]. 
This narrative analysis attempts to illustrate the changes following damage in the brain. We have obtained evidence from the clinical trials and studies done during the last decade. We used the keywords such as concussion and epilepsy, brain damage and seizure, head injury and epilepsy, post-traumatic epilepsy, motor vehicle crash, and epilepsy, post-traumatic to gather data from the PubMed database. The study aims to explain the particular physiological changes in the body after brain injury and how it reacts to those changes. Trauma can lead to neuronal hyperexcitability and seizures. A thorough understanding of the pathophysiology will decrease the mortality and morbidity following post-traumatic seizures. Thus, minimizing the burden on the healthcare system. Moreover, this will also alleviate the issues that patients and their families face.

\section{Review}

Discussion

The following are the changes that play a vital role in the pathogenesis of PTE.

Post-Traumatic Inflammation

Inflammation plays an exciting role in the pathogenesis of post-traumatic seizures. The three crucial changes that induce convulsions include an increase in HMBP 1, interaction with toll-like receptor-1 (TLR1), interleukin- $\beta$ (IL- $\beta$ ) interaction with its receptors, and increased tumor growth factor- $\beta$ (TGF- $\beta$ ) signaling [14].

Hippocampus showed increased TLR expression after closed concussion injury (CCI) in the experiment conducted on rodents. The increase TLR causes neurogenesis after trauma [15]. After brain insult, alginatederived guluronate oligosaccharide (GOS) enters the macrophages. It activates the TLR-4, which in turn causes protein kinase $\mathrm{B}(\mathrm{Akt})$ phosphorylation and triggers both nuclear factor- $\mathrm{KB}(\mathrm{NF}-\mathrm{KB})$ and mechanistic target of rapamycin (mTOR) (Figure 2) [16]. The mTOR pathway, in turn, causes epilepsy. Guo et al. conducted a randomized controlled study by giving animals rapamycin that inhibited the mTOR and studied its subsequent effect. They found that rapamycin decreases PTE's risk, usually occurring ten wks after the primary insult [17].

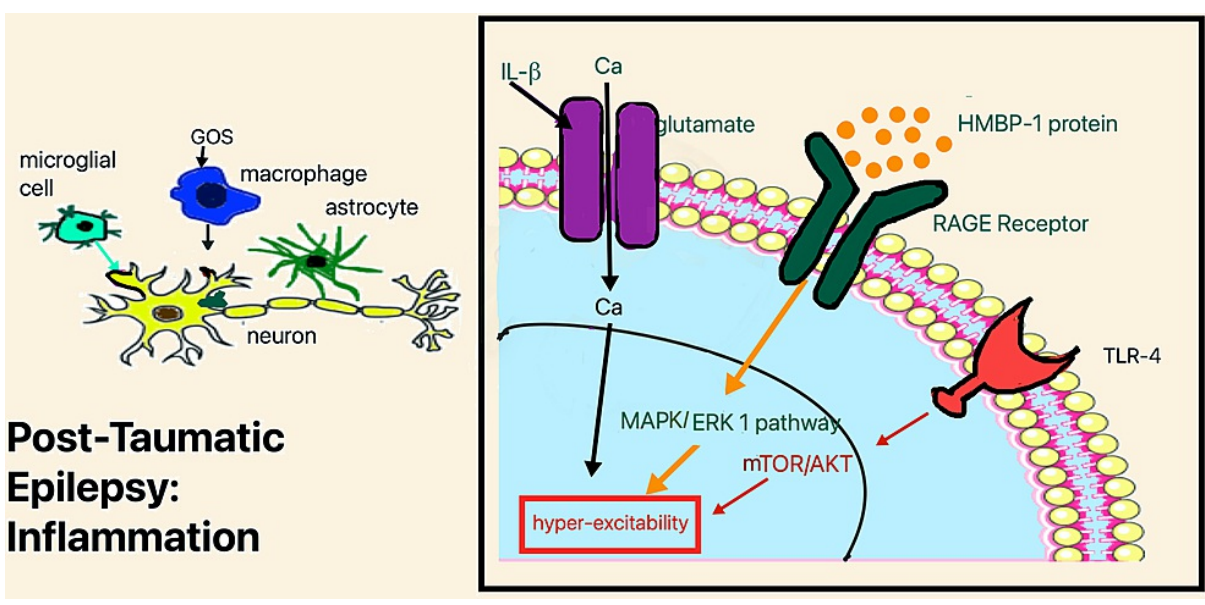

FIGURE 2: Inflammatory changes after trauma: role of TLR, HMBP1 protein, and IL- $\beta$.

TLR: toll-like receptor, HMBP1 protein: high-mobility group box 1 protein, IL- $\beta$ : interleukin- $\beta$, MAPK/ERK 1 pathway: mitogen-activated protein kinase/extracellular signal-related kinase 1, RAGE: receptor for advanced glycation end-products, mTOR/AKT: mechanistic target of rapamycin/protein kinase B, GOS: guluronate oligosaccharide

Prostaglandin E2 (PGE2) expression also increases after injury. Brain permeable compound TG6-10-1 blocks the action of PGE2 and decreases the post-traumatic inflammatory changes and cytokines expression in the brain after injury [18]. Various microglial cells produce IL- $\beta$ and lead to N-methyl-D-aspartate (NMDA) receptor activation (Figure 2). In turn, NMDA activation causes neuronal hyperexcitability via the glutamate pathway by increasing calcium influx in cells. Hyperexcitability can eventually lead to neurodegeneration [19]. Disruption of BBB allows albumin to enter the brain. Astrocytes also uptake that albumin and, as a result, activate the transforming growth factor- $\beta$ /activin receptor-like kinase 5 (TGF- $\beta$ /ALK 5 pathway) [5].

HMBP 1 also causes activation of inflammatory cascade via astrocytes and the transmembrane receptor called receptor for advanced glycation end-products (RAGE). It led to the activation of the mitogen-activated 
protein kinase/extracellular signal-related kinase 1/2 (MAPK/ERK1/2) pathway (Figure 2) and induced astrocyte COX-2. Pedrazzi et al. blocked RAGE via anti-RAGE antibodies and observed a decrease in inflammation [20].

\section{Structural changes after traumatic brain injury}

Traumatic brain injury leads to specific structural changes in the brain. These include the below changes.

BBB Changes

To confirm the changes in BBB after TBI, Tomkins et al. performed a clinical study on 37 post-traumatic patients. All of them had Glasgow Coma Scale (GCS) more than 13 and were seizure-free one week after significant insult. They took images using a Tesla machine to check the BBB integrity and compared them before and after injecting contrast medium Magnetol. They quantified the BBB disruption. Additionally, they documented cortical dysfunction using standard low-resolution brain electromagnetic tomography (sLORETA). They observed slow-wave activity in the brain region with disrupted BBB. They confirmed that the more significant BBB disruption is associated with greater cortical dysfunction and more chances of aberrant slow waves. Subsequently, increasing the risk of PTE [10].

\section{Astrocyte Changes}

The astrocytes respond to the injury, trying to seal the damaged portion from the necrotic tissue by forming scar tissue. astrocytes reach the site of injury and create a scar, thus protecting the rest of the tissue. Anderson et al. conducted an interventional study by inhibiting scar formation in rats after spinal cord injury, and thus the cells fail to regenerate. Then they injected some hydrogel depots of growth factors released by astrocytes and documented the scar formation and regeneration of neurons [5,21].

Type of Injury and Seizure

The study on rodents has previously shown changes in the cortex, hippocampus, thalamus, and amygdala associated with an increased risk of Post-traumatic seizures [22]. Tubi et al. recently performed a longitudinal study on humans to check the percentage of trauma patients developing seizures depending on the area of the brain involved. They followed up a cohort of 90 patients for early seizure outcome and the followed 46 patients longitudinally. They performed a prospective cEEG and high-resolution MRI scan to localize lesions. Surprisingly, $75 \%$ of the patients who had early seizures $(n=24)$ showed temporal lobe injury on MRI. Out of the 46 patients followed for PTE, $45.7 \%$ developed PTE within two years; $85.7 \%$ of those who developed PTE also showed hemorrhagic temporal lobe injury on admission. The small study sample and using specifically Asian cohort has added some bias in the study [23].

Lutkenhoff et al. started a prospective study in 2018. They followed 96 patients surviving TBI. They collected imaging data around day 14 after injury and evaluated structural changes in the cortical and subcortical regions. MRI of 57 patients showed subcortical volume loss; 35 had never developed seizures, 14 had early attacks, and eight had late seizure episodes. They also observed cortical ribbon thinning in 46 patients. Among those, 29 remained seizure-free, 10 developed early attacks, and seven showed late seizure activity [24]. Various researchers explain the changes in the brain after injury (Table 1). 


\section{Cureus}

\begin{tabular}{|c|c|c|c|c|c|}
\hline Author & $\begin{array}{l}\text { Year of } \\
\text { Publication }\end{array}$ & $\begin{array}{l}\text { Study } \\
\text { Duration }\end{array}$ & Population & $\begin{array}{l}\text { Sample } \\
\text { Size }\end{array}$ & Outcome \\
\hline $\begin{array}{l}\text { Tomkins } \\
\text { et al. [10] }\end{array}$ & 2011 & $\begin{array}{l}\text { Two } \\
\text { years } \\
(2005- \\
2011)\end{array}$ & $\begin{array}{l}\text { Male and female } \\
\text { age } 27+/-4.2 \text { and } \\
25.3+/-2.8 \text { years }\end{array}$ & 37 & $\begin{array}{l}\text { BBB disruption occurred in } 82.4 \% \text { of PTE patients compared } \\
\text { to } 25 \% \text { of non-epileptic patients. }\end{array}$ \\
\hline $\begin{array}{l}\text { Tubi et al. } \\
\text { [23] }\end{array}$ & 2019 & $\begin{array}{l}\text { (Jan years } \\
\text { 2001- } \\
\text { Dec } \\
\text { 2011) }\end{array}$ & $\begin{array}{l}\text { Caucasian } 73 \text { males } \\
17 \text { females mean } \\
\text { age } 37.7+/-17.83 \\
\text { years }\end{array}$ & 90 & $\begin{array}{l}75 \% \text { of patients who had early seizures and } 85.7 \% \text { of } \\
\text { patients who developed PTE had temporal lobe injury }\end{array}$ \\
\hline $\begin{array}{l}\text { Lutkenhoff } \\
\text { et al. [24] }\end{array}$ & 2020 & 90 days & $\begin{array}{l}\text { Males and females } \\
\text { age } 6-100 \text { years }\end{array}$ & 96 & $\begin{array}{l}14 \text { patients who had an early seizure and eight who had PTE } \\
\text { had subcortical volume loss } 10 \text { patients with early seizures, } \\
\text { and seven with late seizures also showed cortical ribbon } \\
\text { thinning. }\end{array}$ \\
\hline
\end{tabular}

TABLE 1: Changes in the brain of patients with seizures and epilepsy after trauma.

BBB: blood-brain barrier, PTE: post-traumatic epilepsy

Biochemical and genetic associations with post-traumatic epilepsy

The following are the several biochemical and genetic changes contributing to PTE.

Role of Glycolysis

TBI causes significant metabolic changes, and these changes can be a cause of PTE. Vielhaber et al. demonstrated this in 16 known cases of temporal lobe epilepsy. They used high-resolution respirometry to demonstrate the glucose utilization in the hippocampus. Further, 60-90\% of patients during epilepsy showed temporal lobe hypometabolism on [18F] fluorodeoxyglucose positron emission tomography (FDG_PET). The hypometabolism was more in the patients with severe mesial temporal sclerosis [25]. Neural glutamate receptor gene variation was an associated risk factor leading to hyperexcitability after TBI. Of these changes, single nucleotide polymorphism (SNP) in the SLC1A1 gene was most strongly associated with PTE [13].

Increased glucose metabolism after TBI can lead to neuronal hyperexcitability. Koenig et al. conducted a clinical study to support the hypothesis. They utilized the controlled cortical injury CCI model of brain contusion in rats. They used 2-deoxyglucose 2-DG, which is an inhibitor of glycolysis. They first used 2-DG on the cortical brain section in vitro to decrease the epileptiform activity in the brain in mice. During the next step, they treated mice after CCI with 2-DG for one week after injury. Both in vitro and in vivo studies showed a decrease in brain excitability in 2-DG treated rats. Even a ketogenic diet has been tried after TBI to reduce the risk of PTE $[26,27]$.

\section{Changes in Purines and Protein Metabolism}

Adenosine is neuroprotective. However, TBI increases the 2',3' cyclic adenosine monophosphate (2',3' cAMP). 2',3' cAMP triggers neuronal apoptosis, necrosis, and autophagy. 2', $3^{\prime}$-cyclic nucleotide $3^{\prime}$-phosphodiesterase (CNPase) metabolizes 2',3' cAMP to 2'-AMP and 3'-AMP which are subsequently degraded to neuroprotective adenosine. 2',3' cAMP and its metabolites decrease lipopolysaccharide-induced TNF- $\alpha$ and CXCL 10 production by activating the A2A-receptor. Adenosine also suppresses TNF- $\alpha$ and CXCL 10 production by microglial cells in the brain. Determining the protein biomarkers raised after TBI can also help understand the pathogenesis and prevention of PTE $[8,28,29]$.

Newell-Rogers et al. explained the role of astrocytes and peripheral lymphocytes activation in epileptogenesis. Macrophages cause neuroinflammation, which plays a vital role in the pathogenesis of PTE. The cytokine macrophage migration inhibitory factor (MIF) increases in the brain after TBI. MIF can affect the hippocampus neuronal activity, lowering the threshold of PTE. Inhibiting MIF interaction with the cluster of differentiation 74 (CD 74) by using MIF antagonist (S, R)3-(4-hydroxyphenyl)-4,5-dihydro-5isoxazole acetic acid methyl ester (ISO1) resulted in decreased astrocytosis. However, ISO1 couldn't prevent brain degeneration. The Fluid Percussion injury model was used in mice and injected ISO 130 minutes after an injury to observe the results. Moreover, to explain the role of ISO 1 in gut lymphocytes activation they, checked the $ү \delta \mathrm{T}$ cells in the gut after trauma. They compared before and after levels of $\mathrm{\gamma} \delta \mathrm{T}$ cells after ISO1 
injection. The $ү \delta \mathrm{T}$ cells were low after ISO1 therapy [30].

\section{Post cranioplasty seizures}

Decompression (DC) surgery lowers the raised Intracranial pressure after trauma, and cranioplasty reconstructs the brain. Cranioplasty also lowers the threshold of convulsions. Spencer et al. conducted a systematic review. It compared eight previous studies, including 919 cranioplasty patients. They established the link between various factors leading to post craniotomy seizures (Table 2). The pooled incidence of postcranioplasty seizures was $5.1 \%$ with $95 \%$ CI (2.6-8.2) [31].

\begin{tabular}{|c|c|c|}
\hline Risk Factor & Odds Ratio $(95 \% \mathrm{Cl})$ & P-Value \\
\hline Increasing age & 6.1 & 0.006 \\
\hline Contusion at cranioplasty site & 4.8 & 0.015 \\
\hline Use of monopolar diathermy at cranioplasty & 3.5 & 0.04 \\
\hline High DC- cranioplasty interval & - & 0.06 \\
\hline
\end{tabular}

TABLE 2: Factors affecting post-cranioplasty seizures.

DC: decompression

\section{Limitations}

The post-traumatic events leading to seizures include inflammation by cytokines and astrocytes. The role of astrocytes is still controversial and needs to be studied. Most of the studies mentioned in this narrative review are animal-based. Tubi et al. used a small Caucasian cohort in their clinical trial. The high attrition rate might have caused the bias [23].

\section{Conclusions}

Cases of brain injuries are continually growing with the increase in road traffic accidents. Trauma can lead to various outcomes from simple seizures and debilitating post-traumatic epilepsy. Multiple changes occur after a head injury that can lead to these attacks. Researchers globally are actively searching for new ways to explore the events leading to these convulsions. The inflammatory changes that cause PTE and seizures include upregulation of the mTOR pathway via TLR-1. HMBP-1 protein activates the MAPK/ERK 1/2 pathway. NMDA receptor activation increases neuronal hyperexcitability. Systemic changes following trauma include BBB disturbance, cortical volume reduction. Temporal lobe injury is more associated with convulsions than other parts of the brain. The glutamate receptors gene mutation also lowers the threshold of PTE. The increased glucose metabolism after trauma also leads to neuronal hyperexcitability. Adenosine is neuroprotective, which may prevent inflammation as nerves lose neuroprotective adenosine after trauma.

Despite all the research, the pathogenesis of seizures after trauma is still not completely understood. Some people have crippling chronic epilepsy, while others even lose their lives due to acute convulsions. No known anti-epileptic drug is there that can be safely relied on to prevent or treat these episodes. Most of the studies we discussed in this narrative review are animal-based. Randomized controlled trials, placebocontrolled with a large human population, should be conducted to determine the PTE's exact cause.

\section{Additional Information}

\section{Disclosures}

Conflicts of interest: In compliance with the ICMJE uniform disclosure form, all authors declare the following: Payment/services info: All authors have declared that no financial support was received from any organization for the submitted work. Financial relationships: All authors have declared that they have no financial relationships at present or within the previous three years with any organizations that might have an interest in the submitted work. Other relationships: All authors have declared that there are no other relationships or activities that could appear to have influenced the submitted work.

\section{References}

1. GBD 2016 Neurology Collaborators: Global, regional, and national burden of neurological disorders, 19902016: a systematic analysis for the Global Burden of Disease Study 2016. Lancet Neurol. 2019, 18:459-480. 10.1016/S1474-4422(18)30499-X

2. DeGrauw X, Thurman D, Xu L, Kancherla V, DeGrauw T: Epidemiology of traumatic brain injury-associated 
epilepsy and early use of anti-epilepsy drugs: an analysis of insurance claims data, 2004-2014. Epilepsy Res. 2018, 146:41-49. 10.1016/j.eplepsyres.2018.07.012

3. Ferguson PL, Smith GM, Wannamaker BB, Thurman DJ, Pickelsimer EE, Selassie AW: A population-based study of risk of epilepsy after hospitalization for traumatic brain injury. Epilepsia. 2010, 51:891-898. 10.1111/j.1528-1167.2009.02384.x

4. Sharma R, Leung WL, Zamani A, O'Brien TJ, Casillas Espinosa PM, Semple BD: Neuroinflammation in posttraumatic epilepsy: pathophysiology and tractable therapeutic targets. Brain Sci. 2019, 9:318. 10.3390/brainsci9110318

5. Xu S, Sun Q, Fan J, et al.: Role of astrocytes in post-traumatic epilepsy . Front Neurol. 2019, 10:1149. 10.3389/fneur.2019.01149

6. Dadas A, Janigro D: Breakdown of blood brain barrier as a mechanism of post-traumatic epilepsy . Neurobiol Dis. 2019, 123:20-26. 10.1016/j.nbd.2018.06.022

7. Koenig JB, Dulla CG: Dysregulated glucose metabolism as a therapeutic target to reduce post-traumatic epilepsy. Front Cell Neurosci. 2018, 12:350. 10.3389/fncel.2018.00350

8. Jackson EK, Boison D, Schwarzschild MA, Kochanek PM: Purines: forgotten mediators in traumatic brain injury. J Neurochem. 2016, 137:142-153. 10.1111/jnc.13551

9. Danzer SC: A hit, a hit-a very palpable hit: mild TBI and the development of epilepsy . Epilepsy Curr. 2019, 19:261-263. 10.1177/1535759719854758

10. Tomkins O, Feintuch A, Benifla M, Cohen A, Friedman A, Shelef I: Blood-brain barrier breakdown following traumatic brain injury: a possible role in posttraumatic epilepsy. Cardiovasc Psychiatry Neurol. 2011, 2011:765923. 10.1155/2011/765923

11. Jarero-Basulto JJ, Gasca-Martínez Y, Rivera-Cervantes MC, Ureña-Guerrero ME, Feria-Velasco AI, BeasZarate C: Interactions between epilepsy and plasticity. Pharmaceuticals (Basel). 2018, 11:17. 10.3390/ph11010017

12. Kim SY, Senatorov VV Jr, Morrissey CS, et al.: TGF $\beta$ signaling is associated with changes in inflammatory gene expression and perineuronal net degradation around inhibitory neurons following various neurological insults. Sci Rep. 2017, 7:7711. 10.1038/s41598-017-07394-3

13. Ritter AC, Kammerer CM, Brooks MM, Conley YP, Wagner AK: Genetic variation in neuronal glutamate transport genes and associations with posttraumatic seizure. Epilepsia. 2016, 57:984-993. 10.1111/epi.13397

14. Webster KM, Sun M, Crack P, O'Brien TJ, Shultz SR, Semple BD: Inflammation in epileptogenesis after traumatic brain injury. J Neuroinflammation. 2017, 14:10. 10.1186/s12974-016-0786-1

15. Ye Y, Xu H, Zhang X, Li Z, Jia Y, He X, Huang JH: Association between toll-like receptor 4 expression and neural stem cell proliferation in the hippocampus following traumatic brain injury in mice. Int J Mol Sci. 2014, 15:12651-12664. 10.3390/ijms150712651

16. Fang W, Bi D, Zheng R, et al.: Identification and activation of TLR4-mediated signalling pathways by alginate-derived guluronate oligosaccharide in RAW264.7 macrophages. Sci Rep. 2017, 7:1663. 10.1038/s41598-017-01868-0

17. Guo D, Zeng L, Brody DL, Wong M: Rapamycin attenuates the development of posttraumatic epilepsy in a mouse model of traumatic brain injury. PLoS One. 2013, 8:64078. 10.1371/journal.pone.0064078

18. Jiang J, Yu Y, Kinjo ER, Du Y, Nguyen HP, Dingledine R: Suppressing pro-inflammatory prostaglandin signaling attenuates excitotoxicity-associated neuronal inflammation and injury. Neuropharmacology. 2019, 149:149-160. 10.1016/j.neuropharm.2019.02.011

19. Viviani B, Bartesaghi S, Gardoni F, et al.: Interleukin-1beta enhances NMDA receptor-mediated intracellular calcium increase through activation of the Src family of kinases. J Neurosci. 2003, 23:86928700. 10.1523/JNEUROSCI.23-25-08692.2003

20. Pedrazzi M, Patrone M, Passalacqua M, et al.: Selective proinflammatory activation of astrocytes by highmobility group box 1 protein signaling. J Immunol. 2007, 179:8525-8532. 10.4049/jimmunol.179.12.8525

21. Anderson MA, Burda JE, Ren Y, et al.: Astrocyte scar formation aids central nervous system axon regeneration. Nature. 2016, 532:195-200. 10.1038/nature17623

22. Shultz SR, Cardamone L, Liu YR, et al.: Can structural or functional changes following traumatic brain injury in the rat predict epileptic outcome?. Epilepsia. 2013, 54:1240-1250. 10.1111/epi.12223

23. Tubi MA, Lutkenhoff E, Blanco MB, et al.: Early seizures and temporal lobe trauma predict post-traumatic epilepsy: a longitudinal study. Neurobiol Dis. 2019, 123:115-121. 10.1016/j.nbd.2018.05.014

24. Lutkenhoff ES, Shrestha V, Ruiz Tejeda J, et al.: Early brain biomarkers of post-traumatic seizures: initial report of the multicentre epilepsy bioinformatics study for antiepileptogenic therapy (EpiBioS4Rx) prospective study. J Neurol Neurosurg Psychiatry. 2020, 91:1154-1157. 10.1136/jnnp-2020-322780

25. Vielhaber S, Von Oertzen JH, Kudin AF, et al.: Correlation of hippocampal glucose oxidation capacity and interictal FDG-PET in temporal lobe epilepsy. Epilepsia. 2003, 44:193-199. 10.1046/j.15281157.2003.38102.x

26. Koenig JB, Cantu D, Low C, et al.: Glycolytic inhibitor 2-deoxyglucose prevents cortical hyperexcitability after traumatic brain injury. JCI Insight. 2019, 5:126506. 10.1172/jci.insight.126506

27. Koenig JB, Dulla CG: Dysregulated glucose metabolism as a therapeutic target to reduce post-traumatic epilepsy. Front Cell Neurosci. 2018, 12:350. 10.3389/fncel.2018.00350

28. Agoston DV, Kamnaksh A: Protein biomarkers of epileptogenicity after traumatic brain injury . Neurobiol Dis. 2019, 123:59-68. 10.1016/j.nbd.2018.07.017

29. Newell EA, Exo JL, Verrier JD, et al.: 2',3'-cAMP, 3'-AMP, 2'-AMP and adenosine inhibit TNF- $\alpha$ and CXCL10 production from activated primary murine microglia via A2A receptors. Brain Res. 2015, 1594:27-35. 10.1016/j.brainres.2014.10.059

30. Newell-Rogers MK, Rogers SK, Tobin RP, Mukherjee S, Shapiro LA: Antagonism of macrophage migration inhibitory factory (MIF) after traumatic brain injury ameliorates astrocytosis and peripheral lymphocyte activation and expansion. Int J Mol Sci. 2020, 21:7448. 10.3390/ijms21207448

31. Spencer R, Manivannan S, Sharouf F, Bhatti MI, Zaben M: Risk factors for the development of seizures after cranioplasty in patients that sustained traumatic brain injury: a systematic review. Seizure. 2019, 69:11-16. 10.1016/j.seizure.2019.03.014 